Classification

Physics Abstracts

$64.60-68.90-76.60$

\title{
Diffusion coefficients of fluctuations in semi-infinite ferromagnets
}

\author{
W. Korneta \\ Department of Physics, Technical University, Malczewskiego 29, Radom, Poland
}

and Z. Pytel

Institute of Physics of the Polish Academy of Sciences, Al. Lotników 32/46, Warszawa, Poland

(Reçu le 6 juin 1983, accepté le 10 août 1983)

\begin{abstract}
Résumé. - Nous appliquons le modèle d'Ising avec interactions entre proches voisins au cas d'un ferromagnétique semi-infini, les interactions en surface pouvant être différentes de celles en volume. Les fluctuations d'aimantation au voisinage de transitions du deuxième ordre sont décrites par une équation de diffusion contenant un terme d'amortissement. Le coefficient de diffusion des fluctuations paramagnétiques est obtenu en champ moyen pour les transitions en volume, en surface-volume et en surface et nous discutons leur variation avec la distance à la surface et la température.

Abstract. - The nearest-neighbour Ising model of a semi-infinite ferromagnet in which nearsurface couplings may differ from their bulk values is considered. In the critical region near secondorder phase transitions the decay of fluctuations of the magnetization density is described by a diffusion equation with a damping term. Using mean-field theory, the diffusion coefficients of fluctuations in the paramagnetic phase near the ordinary, surface-bulk and surface phase transitions are obtained and their dependence on the distance from the surface of the ferromagnet and on the temperature is discussed.
\end{abstract}

We consider the nearest-neighbour simple cubic Ising model of a semi-infinite ferromagnet. The coordinates of a lattice site are denoted by $(i j)$ where $i$ denotes the number of the two-dimensional layer parallel to a surface of the ferromagnet whereas $\mathbf{j}$ is a two-dimensional vector within each layer. We denote by $K_{i i^{\prime}}$ the nearest-neighbour exchange interaction between spins in layers $i$ and $i^{\prime}$ divided by the Boltzmann constant and the temperature. It equals $K(1+D)$ when both nearest neighbours are situated in a surface layer and $K$ for all other nearest-neighbour pairs. In the critical region near a continuous phase transition the decay of fluctuations of the magnetization density $\bar{m}_{i \mathrm{j}}(t)$ at a position $(i \mathrm{j})$ is described by a diffusion equation with a damping term. In the paramagnetic phase this equation can be written in the following form [1]

$$
\frac{\partial \bar{m}_{i \mathrm{j}}(t)}{\partial t}=\frac{1}{T_{i}}\left(-\bar{m}_{i \mathrm{j}}(t)+\frac{1}{2} \sum_{i^{\prime} \mathbf{j}^{\prime}} K_{i i^{\prime}} \bar{m}_{i^{\prime} \mathbf{j}^{\prime}}(t)\right)
$$

where the summation runs over the nearest neighbours of a position ( $i j)$ and $T_{i}$ denotes the spin lattice relaxation time in the $i$-th layer. The model of a semi-infinite ferromagnet has been 
studied using mean-field theory [2-4], the renormalization-group approach [5-7] and numerical techniques [3]. In this paper we apply mean-field theory to obtain the diffusion coefficients of fluctuations in the paramagnetic phase near the ordinary, surface-bulk and surface phase transitions. In mean-field theory the diffusion coefficients depend on the distance from the surface of the semi-infinite ferromagnet and on the temperature. Mean-field theory often produces a correct qualitative picture and using this theory almost all quantities of interest can be calculated analytically.

We denote by $\Lambda_{i}$ the diffusion coefficient of the fluctuations in the $i$-th layer. It describes a diffusion of the magnetization density in the layer. We apply to $\Lambda_{i}$ the self-consistent procedure proposed by Kawasaki $[8,9]$ for the diffusion coefficient of fluctuations in an infinite threedimensional ferromagnet in the paramagnetic phase. We obtain, as in [10], that $\Lambda_{i}$ is proportional to $\left(L_{i} / \chi_{i}\right)^{1 / 2}$ where $L_{i}$ denotes the autocorrelation time in the $i$-th layer divided by $T_{i}$ whereas $\chi_{i}$ is the magnetic susceptibility in the $i$-th layer. In order to obtain $L_{i}$, let us consider the Ising model of ferromagnetic film with both surfaces the same as the surface of the considered semi-infinite ferromagnet. Following the procedure described in [1] and finding the limit of obtained formulae for the thickness of the film tending to infinity we obtain

$$
L_{i}=\frac{1}{\operatorname{sh} x}+\frac{\mathrm{e}^{-2 i x}}{\left(1-4 D \mathrm{e}^{-x}\right)}\left(\frac{4 D \mathrm{e}^{x}-1}{\operatorname{sh} x}-\frac{\mathrm{e}^{2 x}-1}{\operatorname{sh} x+2}\right)
$$

where $x$ is determined by the following equation

$$
\operatorname{ch} x=K^{-1}-2 \text {. }
$$

The magnetic susceptibility $\chi_{i}$ in the semi-infinite ferromagnet, defined as a derivative of the magnetization in the $i$-th layer with respect to the external uniform magnetic field, can be written in the form [4]

$$
\chi_{i} \sim \frac{1}{\operatorname{sh}^{2}\left(\frac{x}{2}\right)}\left(1+\frac{4 D-1}{\left(1-4 D \mathrm{e}^{-x}\right)} \mathrm{e}^{-i x}\right)
$$

The magnetic susceptibility $\chi_{i}$ tends to infinity for $x$ tending to zero which determines the ordinary phase transition line and the surface-bulk phase transition point or for $\left(1-4 D \mathrm{e}^{-x}\right)$ tending to zero which determines the surface phase transition line. We denote by $T_{\mathrm{c}}$ the temperature of a phase transition and by $\epsilon$ the reduced temperature defined as $T / T_{\mathrm{c}}-1$.

The diffusion coefficient of fluctuations $\Lambda_{i}$ depends on two quantities $L_{i}$ and $\chi_{i}$ which have different critical behaviours. The magnetic susceptibility $\chi_{i}$ depends on all correlations in a system [4] whereas $L_{i}$ depends only on correlations in the $i$-th layer [1] so, in any layer of the semi-infinite ferromagnet, the critical behaviour of $\chi_{i}$ begins at temperatures farther from $T_{c}$ than the critical behaviour of $L_{i}$. The critical behaviour of $\Lambda_{i}$ at temperatures farther from $T_{\mathrm{c}}$ is thus determined by the critical behaviour of $\chi_{i}$ whereas at temperatures very near $T_{\mathrm{c}}$ it is mainly determined by the critical behaviour of $L_{i}$.

The diffusion coefficients in layers near the surface of semi-infinite ferromagnets with different values of $D$ are shown in figure 1 . According to the value of $D$, curves in this figure show the behaviour of the diffusion coefficients near the ordinary $(D<1 / 4)$, the surface-bulk $(D=1 / 4)$ or the surface $(D>1 / 4)$ phase transition. In the critical region near any phase transition the diffusion coefficients of fluctuations decrease when the temperature tends to $T_{\mathrm{c}}$. The ordinary phase transition is induced by all interactions in the ferromagnet whereas the surface phase transition is induced only by large surface interactions so the diffusion coefficients of fluctuations near the ordinary and the surface phase transitions behave differently. At temperatures near 

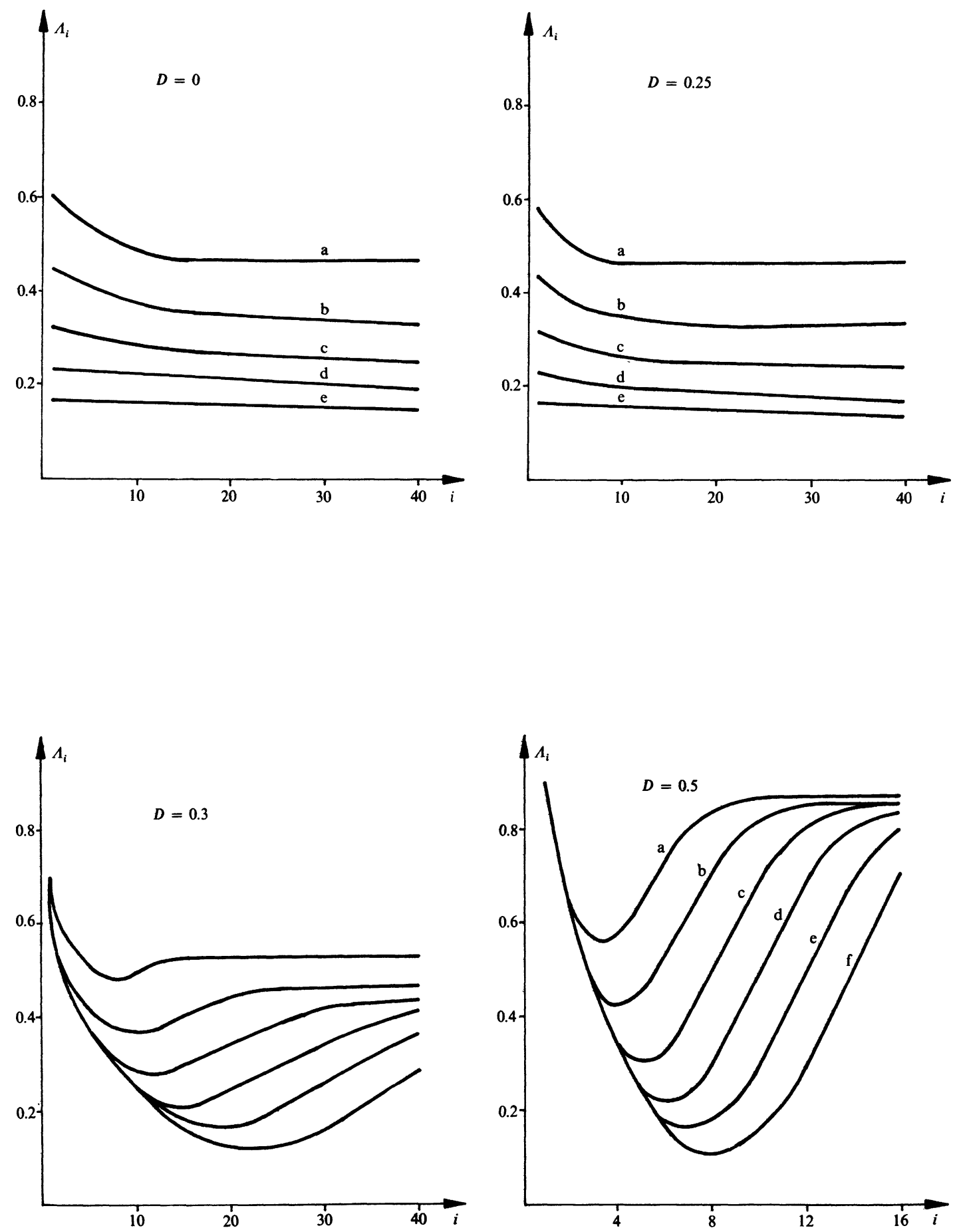

Fig. 1. - The diffusion coefficients of fluctuations $\Lambda_{i}$ in layers near the surface of semi-infinite ferromagnets with different values of $D$ for reduced temperatures $2^{-7}(\mathrm{a}), 2^{-9}(\mathrm{~b}), 2^{-11}(\mathrm{c}), 2^{-13}(\mathrm{~d}), 2^{-15}(\mathrm{e})$ and $2^{-17}$ (f). 
the ordinary phase transition, the diffusion coefficients in different layers far from the surface of the semi-infinite ferromagnet are equal and do not depend on $D$. They slightly increase in layers near the surface over a distance which approximately equals the average correlation length. At temperatures near the surface phase transition, the diffusion coefficients in layers far from the surface of the ferromagnet are determined by the extraordinary phase transition. In layers near the surface, correlations of a spin with spins on the surface dominate and they determine the critical behaviour of physical quantities. The critical behaviour of $\chi_{i}$ induced by large surface interactions begins in layers farther from the surface of the ferromagnet than the critical behaviour of $L_{i}$ because $\chi_{i}$ contains correlations with the surface, whereas $L_{i}$ contains only correlations within the layer. Therefore, at temperatures near the surface phase transition temperature, when we approach the surface of the ferromagnet, $\Lambda_{i}$ first decreases because $\chi_{i}$ quickly increases whereas very near the surface $\Lambda_{i}$ increases because $L_{i}$ quickly increases. In layers farther from the surface of the semi-infinite ferromagnet where the diffusion coefficients of fluctuations decrease, all correlations determine the critical behaviour of physical quantities. In layers near the surface where the diffusion coefficients increase, the nearest correlations dominate and determine the critical behaviour of physical quantities.

In systems with surfaces it is necessary to define a certain number of new critical exponents which refer to local physical quantities [3]. We define the critical exponent $w\left(\Lambda_{i}\right)$.of the diffusion coefficient of fluctuations $\Lambda_{i}$ as the derivative of $\operatorname{Ln} \Lambda_{i}$ with respect to $\operatorname{Ln} \in$. Critical exponents $w\left(\Lambda_{i}\right)$ in the range of reduced temperatures between $2^{-7}$ and $2^{-15}$ in some layers of semiinfinite ferromagnets with different values of $D$ are shown in figure 2 . When $\in$ decreases, the critical exponent $w\left(\Lambda_{i}\right)$ in any layer farther from the surface of the semi-infinite ferromagnet changes from the value like that in an infinite three-dimensional ferromagnet to the value like that in the surface layer. At temperatures farther from $T_{\mathrm{c}}$ the exponent $w\left(\Lambda_{i}\right)$ changes like $w\left(\chi_{i}\right)$ because $w\left(L_{i}\right)$ is constant whereas at temperatures near $T_{\mathrm{c}}$ it changes like $w\left(L_{i}\right)$ because $w\left(\chi_{i}\right)$ is already constant. In the semi-infinite ferromagnet with $D$ equal to zero, the critical exponent $w\left(\Lambda_{i}\right)$ equals 0.25 at temperatures very far and very near $T_{\mathrm{c}}$. In a certain range of temperatures it however tends to another value because $w\left(\chi_{i}\right)$ and $w\left(L_{i}\right)$ change in different ranges of temperatures. In the semi-infinite ferromagnet with a great value of $D$, the exponent $w\left(\Lambda_{i}\right)$ at temperatures far from the surface phase transition temperature $T_{\mathrm{c}}$ equals zero and it increases at temperatures where $\chi_{i}$ dominates. At temperatures very near $T_{\mathrm{c}}$, the quantity $L_{i}$ increases and $w\left(\Lambda_{i}\right)$ in any layer tends to the value $w\left(\Lambda_{1}\right)$ as in the surface layer. The value of $w\left(\Lambda_{1}\right)$ is in this case the same as in an infinite two-dimensional ferromagnet.

In the semi-infinite ferromagnet with $D$ between 0.25 and 0.4 , the distance between the temperatures of the surface and the extraordinary phase transitions is small so the extraordinary phase transition has an effect on the behaviour of critical exponents near the surface phase transition. In such a ferromagnet, the critical exponent $w\left(\Lambda_{i}\right)$ in any layer at temperatures farther from the surface phase transition temperature is determined by the extraordinary phase transition and when $\epsilon$ decreases the exponent calculated with respect to the surface phase transition temperature tends to zero. At temperatures very near the surface phase transition temperature, the term $1 /\left(1-4 D \mathrm{e}^{-x}\right)$ (formulae 2 and 4$)$ is the most important so $w\left(\Lambda_{i}\right)$ behaves as described above in the semi-infinite ferromagnet with a great value of $D$. In the mean-field theory the critical exponent $w\left(\chi_{i}\right)$ equals -1 in an infinite two- and three-dimensional ferromagnet. In the semi-infinite ferromagnet with $D$ equals to 0.25 the changes of $w\left(\Lambda_{i}\right)$ in any layer near the surface-bulk phase transition are thus caused by the changes of the exponent $w\left(L_{i}\right)$. Comparing dependences of $w\left(\Lambda_{i}\right)$ on the reduced temperature in the same layers of semi-infinite ferromagnets with $D$ equals to 0 and 0.25 , it is seen that $w\left(L_{i}\right)$ and $w\left(\chi_{i}\right)$ change in different ranges of temperatures.

We conclude that in the critical region near a second-order phase transition, a physical quantity which consists of some other quantities in a certain range of temperatures has the same 

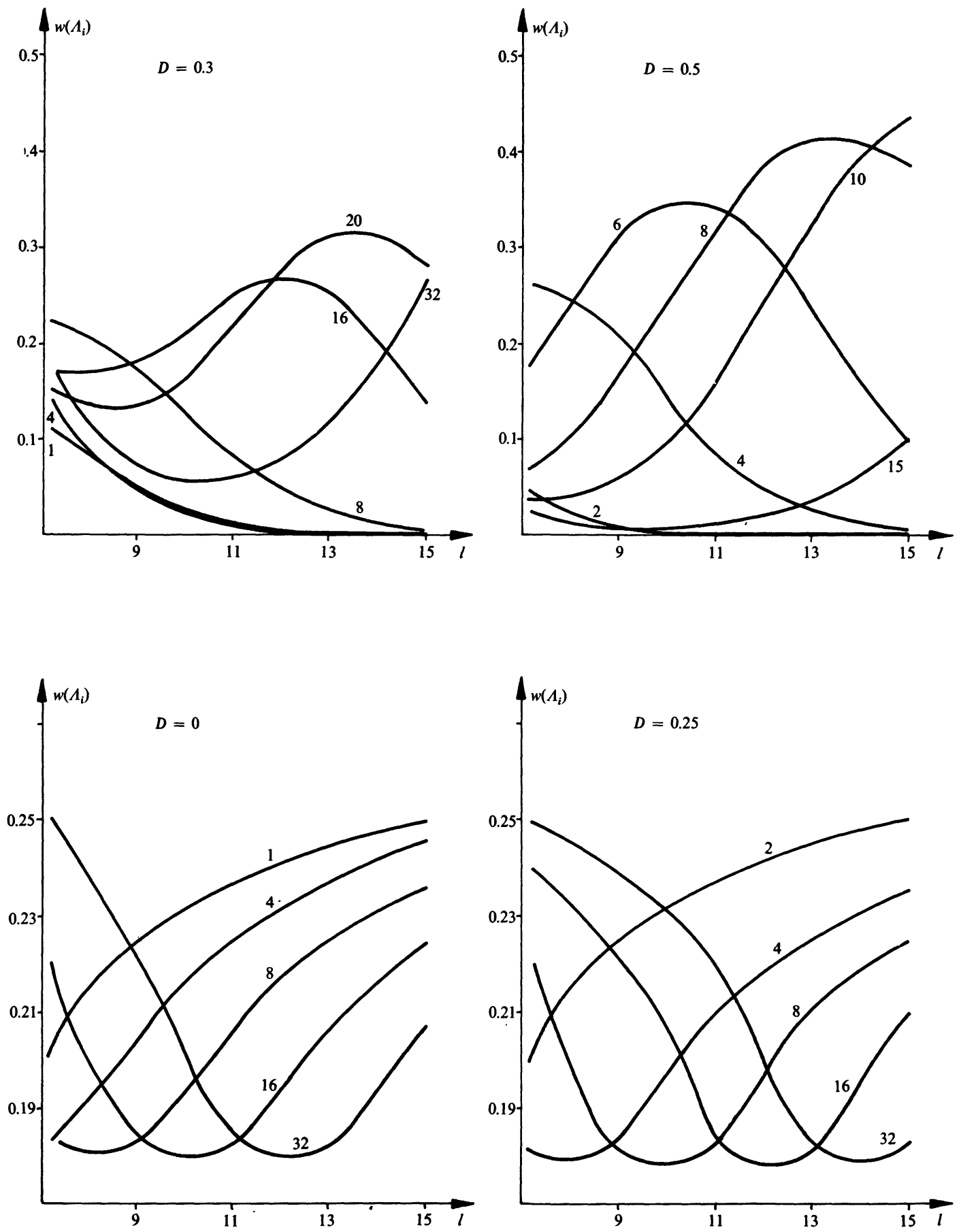

Fig. 2. - Critical exponents of the diffusion coefficients of fluctuations $w\left(\Lambda_{i}\right)$ in some layers of semiinfinite ferromagnets with different values of $D$ in the range of reduced temperatures between $2^{-7}$ and $2^{-15}$. The reduced temperature is $2^{-l}$ where $l$ is on the horizontal axis. 
critical exponent behaviour and satisfies the same scaling relations as does its component quantity whose critical behaviour dominates in this range. In any position of a semi-infinite system at temperatures far from the temperature of a continuous phase transition, the critical behaviours of physical quantities are determined by the phase transition induced by internal interactions in the system. Critical exponents of physical quantities are in this case like those in an infinite three-dimensional system. At temperatures very near a phase transition temperature, the critical behaviours of physical quantities are determined by two-dimensional phase transition induced only by large surface interactions or they are determined by the phase transition induced by both surface and internal interactions. In this case critical exponents of physical quantities in any position of a semi-infinite system are the same as on the surface of the system.

\section{Acknowledgments.}

The authors would like to thank Prof. L. Wojtczak for suggesting the problem. The work is sponsored by the Institute of Physics of the Polish Academy of Sciences.

\section{References}

[1] Korneta, W. and Pytel, Z., Phys. Lett. A 92 (1982) 345.

[2] Mills, D. I., Phys. Rev. B 3 (1971) 3887.

[3] Binder, K. and Hohenberg, P. C., Phys. Rev. B 6 (1972) 3461.

[4] Lubensky, T. C. and Morton, H. R., Phys. Rev. B 12 (1975) 3885.

[5] Lubensky, T. C. and Morton, H. R., Phys. Rev. B 11 (1975) 4533.

[6] Bray, A. J. and Moore, M. H., J. Phys. A 10 (1977) 1927.

[7] Burkhardt, Th. and Eisenriegler, E., Phys. Rev. B 16 (1977) 3213.

[8] Kawasaki, K., J. Phys. Chem. Solids 28 (1967) 1277.

[9] Kawasaki, K., Progr. Theor. Phys. 39 (1968) 285.

[10] Korneta, W. and Pytel, Z., J. Phys. C 15 (1982) L 1099. 